\title{
Essais
}

Revue interdisciplinaire d'Humanités

$11 \mid 2017$

Fictions de l'identité

\section{Pour une approche littéraire de l'identité}

Avant-propos

Magali Fourgnaud

\section{(2) OpenEdition}

Journals

Édition électronique

URL : http://journals.openedition.org/essais/3237

DOI : $10.4000 /$ essais.3237

ISSN : 2276-0970

Éditeur

École doctorale Montaigne Humanités

\section{Édition imprimée}

Date de publication : 15 mai 2017

Pagination : 7-15

ISBN : 979-10-97024-01-7

ISSN : 2417-4211

\section{Référence électronique}

Magali Fourgnaud, « Pour une approche littéraire de l'identité », Essais [En ligne], 11 | 2017, mis en ligne le 14 octobre 2020, consulté le 21 octobre 2020. URL : http://journals.openedition.org/essais/3237 ; DOI : https://doi.org/10.4000/essais.3237 


\title{
Pour une approche littéraire de l'identité
}

\section{Avant-propos}

\section{Magali Fourgnaud}

\begin{abstract}
Il est des époques historiques où la vie individuelle semble s'effacer dans la préoccupation de la vie générale ; mais, si on y regarde de plus près, on voit que, tout au contraire, les préoccupations personnelles prennent une importance d'autant plus grande, aux époques de troubles et d'incertitude, que l'on est surexcité par la vie générale. Ne sont-ce pas les époques fécondes en rêves, en projets, en situations romanesques, en accès d'enthousiasme, de doute et d'effroi ${ }^{1}$ ?
\end{abstract}

Emportée dans l'élan romantique qui fait du théâtre intérieur de l'individu le miroir ou l'écho du monde extérieur, Georges Sand exprime ainsi le rapport intime qui s'instaure selon elle entre les événements historiques et le processus de construction de soi : les périodes de troubles politiques et sociaux déclencheraient ainsi chez l'individu une interrogation sur son passé et sur son avenir, sur ce qui définit sa personnalité, sur la manière dont il se situe par rapport aux autres, en somme un questionnement existentiel et identitaire. Le flot médiatique actuel réitère, sans vraiment l'interroger, ce lien considéré comme évident entre crise politique, économique, sociale et crise identitaire. Tout se passe comme si la définition d'un socle identitaire auquel se référer permettait d'échapper à l'instabilité (sociale, économique, politique) qui menace et d'oublier un temps la gravité des situations vécues par les individus. "Crise " et "identité " se trouvent ainsi enfermées dans un discours incantatoire et performatif qui exprime et transmet des affects collectifs, qu'il convient d'interpréter pour alimenter la compréhension du monde actuel. D'ailleurs, la notion d' «identité » est-elle la plus opérante pour rendre compte, analyser et interpréter les diverses modalités d'être soi et les expressions de soi dans les temps d'incertitudes politiques et économiques? Les recherches en sciences sociales ne doivent-elles pas résister au chant des sirènes médiatiques qui alimentent les peurs et la haine d'autrui ?

1 Dans la préface de 1855 à Le Diable aux champs, George Sand, Préfaces, éd. A. Szabo, Debrecen, Studia Romanica de Debrecen, 1997, 2 vol., vol. I, p. 235, cité par Damien Zanone, Le Moi, l'Histoire, 1789-1848, éd. D. Zanone, Grenoble, ELLUG, Université Stendhal, 2005, p. 7. 
Le présent dossier $^{2}$ vise à voir comment les pratiques discursives, philosophiques et littéraires, témoignent de la variété des appréhensions du " soi ", des manières d'être "soi " et de se dire comme "soi " en période de crise. Cette étude réinterroge ainsi les notions de " crise " et d' "identité ", et surtout remet en question le rapport que l'on a tendance à rapidement établir entre les deux. En effet, pourquoi les périodes d'incertitude (sur les plans politique, économique et social) induiraient-elles davantage un repli identitaire ou une crise d'identité individuelle que les périodes de stabilité ?

Tout d'abord, une enquête terminologique et historique paraît nécessaire : de quoi parle-t-on lorsqu'on utilise le mot "crise "? Contrairement à la perception actuelle du mot, la crise est, à l'origine, le moment du diagnostic (le mot a d'abord un sens médical), puis de la décision, avant le passage à l'action. Conformément à son étymologie (krisis est issu du verbe grec uoíveıv, qui signifie "discerner ", "trier ", à l'origine du mot " critique »), la crise connote l'idée d'un retour réflexif sur soi-même, d'un moment critique propice à la critique, c'est-à-dire à l'usage de sa raison, de sa faculté de juger. C'est de manière significative au XVIII ${ }^{\mathrm{e}}$ siècle que ce sens de la crise comme critique a été stabilisé, comme l'a montré Reinhard Koselleck ${ }^{3}$. La crise, en tant que critique, est ainsi l'expression d'une exigence de ne pas s'en tenir aux faits, aux propositions du passé ; elle témoigne d'un désir de renouveler ce qui est déjà là, de chercher de nouvelles manières d'être au monde et d'être soi, tout en acceptant le principe d'incertitude de la réalisation : "C'est dans la nature de la crise d'être l'échéance d'une décision qu'on attend. Et la décision attendue reste incertaine. Dans l'insécurité générale d'une situation critique, il y a donc cette seule certitude qu'une fin de l'état critique se prépare, sans qu'on sache quand et comment on y parviendra ${ }^{4}$." L'émergence de ce sens de la crise comme critique au XVIII ${ }^{\mathrm{e}}$ siècle souligne son rapport intrinsèque avec la modernité. Telle est la thèse défendue par Myriam Revault d'Allonnes, dans La Crise sans fin, essai sur l'expérience moderne du temps. Pour la philosophe la crise est même consubstantielle à la modernité, celle-ci se définissant par une rupture avec l'Ancien : le propre de la modernité est en effet de trouver

2 Les travaux ici rassemblés sont le fruit d'une réflexion collective débutée au cours de l'année universitaire 2012-2013, au sein l'École Doctorale Montaigne-Humanités, autour du rapport entre troubles politiques et constructions identitaires. Cette recherche collective a été ponctuée par plusieurs temps forts : une table ronde en présence de la philosophe Myriam Revault d'Allonnes, autour de son ouvrage, La Crise sans fin, essai sur l'expérience moderne du temps, puis une journée d'études consacrée aux rapports entre troubles politiques et constructions identitaires, organisée par Marion Bourbon, Magali Fourgnaud et Hugo Remark, avec le soutien de l'École Doctorale et du laboratoire SPH (Sciences, Philosophie, Humanités), le 22 avril 2013.

3 Reinhart Koselleck et Michaela W. Richter, Crisis, in Journal of the History of Ideas, Vol. 67, No. 2 (Apr., 2006), p. 357-400.

4 Reinhard Koselleck, Kritik und Krise, ein Beitrag zur Pathogenese der bürgerlichen [1959], Le Règne de la critique, trad. Han Hildenbrand, Paris, Éditions de Minuit, 1979, p. 107.

5 Voir ci-dessous l'entretien de Myriam Revault d'Allonnes. 
sa normativité en elle-même, en dehors de toute instance supérieure. La crise n'est donc pas simplement la déploration d'un état de faits (" c'est la crise »), elle est le signe d'une remise en question des fondements et un passage à l'acte. Penser la crise comme la remise en question de nos catégories et la relier aux nouveaux modes de subjectivités apparaissentt dès lors comme une nécessité pour faire face aux défis contemporains, élan auquel notre numéro souhaiterait contribuer, en réinterrogeant en particulier le concept d'identité.

Les connotations essentialistes et exclusives que le terme "identité " porte en lui nous invitent à la plus grande prudence. Les sciences sociales ne feraient-elles d'ailleurs pas mieux de se passer de cette notion ? De fait, comme l'a souligné Pierre Nora en 2007, le terme suppose inévitablement la préexistence d'une identité, comme "un fait de substance ${ }^{6}$ ", invariable dans le temps. Il réifie ainsi ce dont il parle, construit le mythe d'une permanence et d'une cohérence autour de ce qui, par définition, échappe à toute clôture, à savoir le devenir de l'individu. Les historiens, semble-t-il, ont été les premiers à remettre en question l'emploi de cette notion, sans doute parce que l'histoire, ou plutôt le récit historiographique, est récupérée de manière récurrente à des fins politiques. Mais cette vision de l'histoire comme roman national est ellemême historique, intimement liée à la Révolution. Rogers Brubaker en conclut qu' " [...] il faut se garder de reproduire ou de conforter involontairement une telle réification, en adoptant, sans esprit critique, des catégories de pratique comme catégories d'analyse $\mathrm{d}^{7}$. "Certes, pour tenter de contrebalancer la conception dogmatique, sectaire et intolérante de l'identité, réduite alors à une seule appartenance, de nombreux auteurs ont défendu l'idée d'une identité plurielle ("identities are robustly plural ", affirme vigoureusement Amartya $\operatorname{Sen}^{8}$ ), changeante, faite de l'ensemble des appartenances qui nous traversent. Or cette conception constructiviste de l'identité, qui vise à adoucir l'essentialisme inhérent à la notion en défendant l'idée d'un moi multiple et métissé, pose elle-même problème : s'il y a dispersion de ce que je suis en plusieurs " moi ", en quoi peut-on encore parler d'identité ? À une plus grande échelle, si on s'attache à reconnaître les différences des individus, pourquoi s'attacher à définir des «identités collectives »? En outre, en parlant d'une " identité " plurielle, on présente comme étant la solution, ce qui est en fait l'énoncé même du problème : dans quelle mesure le mot "identité ", dont le premier sens désigne ce qui, sous des dénominations ou des aspects divers, ne fait qu'un ou ne représente qu'une seule et même réalité, peut-il rendre compte de la complexité et la multiplicité de ce que nous sommes?

6 Pierre Nora, «Les Avatars de l'identité nationale ", Le Débat, 2010/2 (n 159), p. 5.

7 Rogers Brubaker, "Au-delà de l'" identité ", Actes de la recherche en sciences sociales, 2001/4 (nº 139), p. 70.

8 Amartya Sen, Identity and Violence: The Illusion of Destiny [2006], Identité et Violence, trad. S. Kleiman-Lafon, Paris, Odile Jacob, 2010. 
Si le concept d'identité est si problématique, c'est avant tout parce qu'il suppose des manières d'observer le réel résolument incompatibles. Dans les années 1960, par exemple, les sciences sociales, sous l'impulsion d'Erwin Goffman, ont employé le mot "identity " dans un sens opposé à celui d'Erikson (le père de la notion psychologique de "crise d'identité ») : alors que pour ce dernier, avoir plusieurs identités est le signe d'une aliénation mentale, dans le sens interactionniste, l'identité n'est qu'un masque, un rôle social, qu'un individu doit savoir jouer, mais aussi savoir quitter pour passer à un autre. Ces conceptions radicalement opposées mettent en évidence la grande polysémie d'un terme que l'on a vite fait d'employer sans en proposer une définition claire. De fait, le terme a connu une évolution sémantique profonde. Tandis que traditionnellement, le mot « identité " désignait le fait " qu'il n'y a qu'une seule et même chose là où on aurait pu penser qu'il y en avait deux ", le mot en est venu, depuis une dizaine d'années, à signifier autre chose, à savoir « qu'il y a une chose qui possède la vertu d'être elle-même alors qu'elle aurait pu ne pas encore être elle-même ou ne plus être elle-même "', comme le souligne Vincent Descombes. Pour autant, se défaire complètement de l'idiome identitaire nous empêcherait de comprendre ce qui se joue dans l'affirmation et la construction du sujet, lorsqu'il cherche à définir son identité. En effet, ce que je suis c'est ce que j'exprime par mes actes, mais c'est aussi l'histoire dont je suis l'œuvre et les liens sociaux que je tisse. Dès lors, sans nier la dimension imaginaire de l'identité collective, Vincent Descombes lui accorde cependant un "pouvoir instituant ", selon la terminologie de Castoriadis : «[...] l'homme d'aujourd'hui, tout en maintenant que ce droit du sujet est un droit à l'émancipation, l'interprète aussi dans le sens d'un droit à définir lui-même son identité comme il la conçoit, ce qui le conduit à y inclure des liens sociaux qui ne doivent en rien à un contrat social. Il se sert de l'idiome identitaire pour faire un pas en direction d'une réconciliation avec sa propre humanité. [...] Elle l'autorise à dire "moi" pour autre chose que lui-même ${ }^{10}$." Les revendications identitaires seraient ainsi l'expression de la volonté des sujets à se déterminer eux-mêmes : se passer de la notion risque ainsi de nous empêcher d'analyser les processus d'individuation propres à notre modernité.

Dès lors, quand les sciences sociales parlent d' "identité ", ne cherchentelles pas plutôt à analyser les processus d'individuation et de subjectivation? L'anthropologie actuelle remet elle aussi en question la notion d'identité au profit de la notion de "subjectivité » : " La figure du sujet permet de dépasser les problématiques de l'identité qui ont occupé et même obsédé l'anthropologie depuis ses origines ethnicistes jusqu'aux polémiques récentes ${ }^{11}$ ", selon

9 Vincent Descombes, Les Embarras de l'identité, Paris, Gallimard, NRF Essais, 2013, p. 14.

10 Ibid., p. 253.

11 Michel Agier, "Penser le sujet, observer la frontière. Le décentrement de l'anthropologie ", L'Homme 2012/3 (n' 203-204), p. 51. 
Michel Agier. Pour rendre compte des expériences humaines vécues au cours des migrations, sur les zones frontières, dans des espaces de l'entre-deux, les notions de personne (qui renvoie à la composition sociale de l'être humain) ou d'individu (que la sociologie utilise pour établir des classements selon le sexe, l'âge, la catégorie socio-professionnelle...) ne suffisent plus. Mais loin de tomber dans l'écueil de la prétendue définition d'une "vérité du sujet ", l'anthropologue entend " réfléchir aux conditions de l'irruption d'un sujet dans un moment et un lieu où une subjectivation rend le sujet possible, en tenant l'identitaire à distance ${ }^{12}$. » La démarche proposée par Michel Agier est donc une "expérience des concepts en situation ", afin de les travailler et de les transformer, plutôt que de s'appuyer sur une définition théorique préconçue.

Grâce à sa fonction expérimentale et épistémologique, la littérature participe à cette remise en question et à cette expérimentation de nos catégories, en particulier de la notion d'identité. Certes, une veine de la littérature, que l'on serait tenté de nommer "identitaire ", se présente comme la voix des minorités et revendique un rôle politique essentiel dans la mesure où elle permet à certains groupes sociaux d'exister dans l'espace social et d'acquérir une nouvelle forme de représentation. Comme l'écrit Hervé Serry, ces formes littéraires permettent de "donner une forme légitime à ce qui était considéré jusqu'alors comme un stigmate ${ }^{13}$." Tout en reconnaissant la fécondité de ces approches, la perspective adoptée dans ce numéro est différente. Il s'agit moins d'étudier la manière dont la littérature peut participer à une revendication identitaire que de voir comment elle interroge et pense la notion d'identité. En somme, la littérature est considérée ici comme un laboratoire, un lieu d'interrogation et d'expérimentation des modalités de subjectivation. En effet, si l'identité est une fiction ${ }^{14}$, une construction mentale qui tisse des liens entre ce qui, dans le flux de l'expérience humaine, est perçu de manière éparse et discontinue, la littérature permet d'en comprendre les enjeux et les processus de construction. De fait, chacun peut en faire l'expérience : la multiplicité des sensations et des expériences empêche toute réduction du moi à une unité. C'est notre imagination qui construit la cohérence, en établissant des liens entre des éléments disjoints, en transformant des faits en causes et en conséquences. Comme le souligne Paul Ricœur : "Répondre à la question « qui ? »... c'est raconter l'histoire d'une vie. L'histoire racontée dit le qui de

12 Ibid., p. 60.

13 Hervé Serry, "La littérature pour faire et défaire les groupes ", Littératures et identités, Sociétés contemporaines $\mathrm{n}^{\circ} 44,2001, \mathrm{p} .7$.

14 "L'identité que nous attribuons à l'esprit de l'homme n'est qu'une identité fictive, du même genre que celle que nous attribuons aux corps végétaux et animaux. Elle ne peut donc pas avoir une origine différente mais doit provenir d'une opération semblable de l'imagination sur des objets semblables. ", Thomas Hume, A treatise of human nature [1739], Traité de la nature humaine, trad. Philippe Saltel, Livre I, Appendice, Paris, Garnier-Flammarion, 1993, p. 351. 
l'action. L'identité du qui n'est donc elle-même qu'une identité narrative ${ }^{15}$." Dès lors, la littérature, par son pouvoir de déconstruction des fictions, participe au questionnement suscité par l'identité. En mettant en scène le processus de construction identitaire, elle empêche de naturaliser cette catégorie, rendant le lecteur plus critique et plus lucide sur des réflexes individuels ou sociaux qu'il peut observer. En outre, la fiction joue également un rôle de schématisation et de modélisation, au sens cognitif du terme : de la même manière que les philosophes utilisent des fables et autres métaphores pour construire les concepts, de même, la fiction littéraire permet de conceptualiser et d'interroger certaines catégories problématiques, comme l'identité. Ainsi, dans son Essai sur l'entendement humain (1689) ${ }^{16}$, Locke distingue l'individu (le corps matériel de l'être humain) et la personne, qui se définit par la mémoire et la conscience de soi. Il a donc une conception subjective de l'identité. Pour saisir cette différence entre l'individu et la personne, le philosophe anglais développe un récit de métempsycose racontant l'échange de l'âme d'un prince et celle d'un savetier ${ }^{17}$. La fiction sert ici d'expérience de pensée et permet de concevoir des distinctions que la perception quotidienne nous empêche de faire. Dans ses Lettres persanes, Montesquieu a lui aussi recours à un conte à métempsycose, qu'il insère dans un roman arabe inventé par Rica. Le cruel sultan Ibrahim en vient à tuer l'une des femmes de son sérail, Anaïs. Des mondes célestes où elle se retrouve, celle-ci monte un stratagème : échanger l'âme du sultan par celle d'un eunuque doux et bienveillant. Ce tour a pour effet de modifier complètement les règles du sérail et lorsque l'ancien Ibrahim revient, il est considéré comme un étranger et un usurpateur. Le conte littéraire fonctionne ici comme la fable lockéenne : l'apparence corporelle du sultan est bien la même, mais son âme (sa personne) a changé. Ces fictions expérimentales, fréquentes au XVIII ${ }^{e}$ siècle $^{18}$, réaffirment une conception dualiste de l'être humain (conçu comme corps et âme), tout en témoignant de l'émergence du sujet moderne : seul l'individu, dans sa conscience et sa mémoire, peut

15 Paul Ricœur, Temps et Récit, Paris, Seuil-Essais, 1958, t. III, Le Temps raconté, p. 442-443.

16 John Locke, An Essay Concerning Human Understanding [1689], Essai philosophique concernant l'entendement humain, trad. Pierre Coste, éd. P. Hamou, Paris, Librairie générale française, "Classique de la philosophie ", 2009.

17 « [... l'âme d'un prince accompagnée d'un sentiment intérieur de la vie de prince qu'il a déjà menée dans le monde, vint à entrer dans le corps d'un savetier, aussitôt que l'âme de ce pauvre homme aurait abandonné son corps, chacun voit que ce serait la même personne que le prince, uniquement responsable des actions qu'elle aurait faites étant prince. Mais qui voudrait dire que ce serait le même homme? Le corps doit donc entrer aussi dans ce qui constitue l'homme ; et je m'imagine qu'en ce cas-là le corps déterminerait l'homme, au jugement de tout le monde ; et que l'âme accompagnée de toutes les pensées de prince qu'elle avait autrefois, ne constituerait pas un autre homme. Ce serait toujours le même savetier, dans l'opinion de chacun, lui seul excepté. ", ibid., p. 529.

18 Jean-François Perrin, "Soi-même comme multitude, le cas du récit à métempsycose au XVIII' siècle ", Dix-huitième siècle, nº 41, 2009, p. 168-186. 
se définir lui-même. La fiction littéraire s'apparente ainsi à un véritable exercice de pensée philosophique, qui permet à la fois de conceptualiser et d'expérimenter par l'imagination les affres de l'identité.

Or, par cette dimension réflexive et investigatrice, la littérature forme, transforme, in-forme nos subjectivités. En effet, la littérature, par le double mouvement qu'elle suppose d'immersion dans un univers fictionnel et de distance critique induite par la démarche interprétative, participe en profondeur à la construction du sujet : "De même que l'état d'attention me permet d'habiter le geste que je suis en train de faire, de même l'attitude critique me permet de reconnaitre et saisir certains des gestes qui me traversent, pour en tirer l'occasion de faire progresser mon individuation ${ }^{19}$ ", écrit Yves Citton. En somme, analyser et interpréter nos catégories (notamment l'identité) ouvrent à une meilleure connaissance de soi et partant, modifient le rapport à soi : le travail critique travaille le critique, le transforme et redéfinit son identité. Comme le dit Marielle Macé, la lecture va « d'une dynamique d'individuation (celle d'une œuvre) à une autre (celle du sujet qui la reçoit), autrement dit d'un style à un autre style, entre lesquels surviennent des inventions formelles et des puissances d'être ${ }^{20}$ ». Il ne s'agit pas bien sûr simplement de modèles d'existence à suivre (ou à rejeter) : c'est une modification profonde du rapport au réel qu'induit la lecture littéraire. La rencontre avec un texte littéraire, texte qui résiste, dans lequel l'immersion demande souvent un effort d'adaptation, d'accommodement, allégorise, en quelque sorte, la rencontre avec un Autre radical. Le dépaysement à la fois sensible et conceptuel qu' elle induit - ce que Carlo Ginzburg, traduisant Viktor Chklovski ${ }^{21}$, appelle un effet d' " estrangement $^{22} "-$ modifie durablement ma perception du monde et des autres. En modifiant en profondeur le rapport à soi et au monde, la littérature permet ainsi d'échapper au repli sur soi au profit d'un rapport renouvelé à l'Autre.

19 Yves Citton, Gestes d'humanités, Anthropologie sauvage de nos expériences esthétiques, Paris, Armand Colin, 2012, p. 146.

20 Marielle Macé, Façons de lire, manières d'être, Paris, Gallimard, NRF Essais, 2011, p. 92.

21 «Pour ressusciter notre perception de la vie, pour rendre les choses à nouveau sensibles, faire de la pierre une pierre, il existe ce que nous appelons l'art. La fin de l'art est de nous procurer une sensation de la chose, mais une sensation qui soit une vision, et non pas seulement une reconnaissance. Pour parvenir à ce résultat, l'art utilise deux procédés : l'estrangement des choses et la complication des formes, par laquelle il cherche à rendre la perception plus ardue et à en prolonger la durée. En art, la perception est une fin en soi et doit être amplifiée. L'art est le moyen de voir quelque chose devenir ; ce qui a réellement été n’a aucune importance ", Viktor Šklovski, Une teoria della prosa, trad. M. Olsoufieva, Bari, 1966, p. 15-17. Cité par Carlo Ginzburg, À distance, Neuf essais sur le point de vue en histoire, Occhiacci di legno, nove riflessioni, trad. Pierre-Antoine Fabre, Paris, Gallimard, 2001, p. 16.

22 L'Estrangement, retour sur un thème de Carlo Ginzburg, éd. S. Landi, Essais, Revue interdisciplinaire d'Humanités, Hors-série 2013, Pessac, École Doctorale Montaigne-Humanités, 2013. 
Le dossier qui suit aborde les questions des formes d'expressions identitaires selon trois axes, et en premier lieu dans une perspective historique et philosophique. Philippe Cournault montre comment Saint-Augustin parvient à dépasser la question de l'identité communautaire pour lancer les fondements d'une histoire universelle : tandis que La Cité de Dieu a toutes les apparences d'une réponse identitaire (la construction d'une communauté chrétienne) au trouble politique que fut le sac de Rome, l'étude propose de lire l'ouvrage comme un essai d'histoire transcendante, outrepassant toute incarnation politique, fût-elle chrétienne, ainsi que les aléas de l'histoire humaine. Peut-on dès lors se passer de la notion d'identité ? Telle est la question que soulève Sylvia Giocanti dans son étude des Essais de Montaigne. Alors que ce dernier remet en question l'idée d'une identité individuelle substantielle, l'expérience de soi étant celle d'un moi multiple, morcelé et contradictoire, il peut nous aider malgré tout à mieux comprendre ce qui demeure un " désir inextinguible d'identité » et qui est aussi un désir de reconnaissance de cette identité. Mais cette aspiration ne doit pas aboutir à la fusion de l'individu et de la communauté, au risque de conduire à l'aliénation et donc à la négation du sujet lui-même.

Le deuxième axe se propose de voir dans quelle mesure les mythes et leurs réécritures peuvent être considérés comme des modalités des constructions identitaires collectives. Tel est l'objet des travaux d'Igor Fiatti et de Fanny Blin. Le premier s'interroge sur la manière dont certains auteurs autrichiens contemporains remettent en question les mythes fondateurs de leur identité culturelle. La comparaison fructueuse des œuvres de Thomas Bernhard, d'Ingeborg Bachmann et de Robert Menasse permet de mettre en évidence comment ces auteurs, comprimés entre le poids de l'héritage historique et culturel de l'Allemagne et la prégnance du passé nazi sur la conscience collective, parviennent chacun à leur manière à redéployer un récit qui leur est propre. Fanny Blin, quant à elle, confronte différentes réécritures du mythe d'Antigone, dans des œuvres théâtrales espagnoles entre 1939 et 1980 : elle étudie la manière dont le théâtre témoigne des questionnements identitaires propres à une situation politique troublée. La variété des interprétations du mythe sophocléen montre parfaitement comment l'identité, loin de renvoyer à l'unité fixe d'un invariant anhistorique, est le résultat d'une construction sociale contingente, multiple et même contradictoire.

Enfin, le troisième axe porte sur la manière dont le roman s'empare et traite des questions identitaires, d'une part en mettant en scène la crise d'identité de ses personnages, d'autre part par la posture critique et distanciée qu'il induit. Blandine Puel analyse la manière dont s'exprime la crise existentielle que traversent les deux adolescents dans le premier roman d'Alberto Moravia, Gli Indifferenti, alors que la dictature fasciste est à son apogée. Alessandro Leiduan propose quant à lui une réflexion sur la dimension communicationnelle du récit littéraire : au-delà de l'esthétique, l'auteur étudie la configuration 
qui s'établit à la réception d'œuvres narratives en portant une attention particulière à l'attitude ambiguë qu'adoptent les lecteurs quant au caractère fictionnel des énoncés qui constituent ces récits. S’appuyant notamment sur les travaux de Jean-Marie Schaeffer, il développe l'idée selon laquelle tout effet social de la fiction est subordonné à l'adoption d'une attitude de feintise ludique à sa réception; ce n'est que lorsqu'une société feint de croire à une fiction que celle-ci peut fonctionner comme un dispositif de sauvegarde ou de construction des identités sociales. À travers l'étude d'œuvres narratives d'Umberto Eco qui dévient des formes canoniques, l'auteur montre en creux toute l'ambiguïté sociale de la feintise ludique, et présente ainsi la fiction comme réservoir et vecteur potentiel de croyances réelles non assumées à l'échelle d'une société.

Notre dossier se clôt par deux textes inédits. Le premier est un entretien mené par Lia Kurts avec François Rastier qui revient sur la notion d'" aphorisme ", notion centrale dans la pensée de Saussure. À l'opposé de la conception ontologique du langage, qui entend identifier, classer, ordonner le réel, l'aphorisme saisit dans un même élan des dualités. Il se présente à la fois comme un moyen de remettre en question l'illusion des identités déterminées par le langage et comme un mode d'objectivation spécifique. Cette réflexion théorique est suivie par un ensemble d'aphorismes littéraires de François Vaucluse.

En prenant comme support des textes littéraires, les études suivantes montrent comment la littérature contribue à la problématisation d'une notion complexe, l'identité, réservée jusqu'alors à l'histoire, l'anthropologie ou la psychologie. La littérature non seulement met en scène les rapports entre contexte social et politique et processus de subjectivation mais surtout participe à la constitution de subjectivités critiques : en débordant des modes de raisonnement rationnels, en bousculant nos perceptions, la littérature nous ouvre un espace de liberté pour expérimenter et penser à nouveaux frais nos catégories. En ce sens, elle apparaît comme un rempart indispensable contre les Identités meurtrières, car, comme l'écrivait Amin Maalouf, « [...] c'est notre regard qui enferme souvent les autres dans leurs plus étroites appartenances, et c'est notre regard qui peut les libérer ${ }^{23}$."

\section{Magali Fourgnaud}

EA $4574 \mathrm{SPH}$

Université Bordeaux Montaigne magali.fourgnaud@u-bordeaux-montaigne.fr

23 Amin Maalouf, Les Identités meurtrières, Paris, Grasset, 1998, p. 32. 